\title{
Erratum to: Correlation between pathology and neuromelanin MR imaging in Parkinson's disease and dementia with Lewy bodies
}

\author{
Notice of redundant publication
}

\author{
Shinichiro Kitao ${ }^{1} \cdot$ Eiji Matsusue $^{2} \cdot$ Shinya Fujii $^{1} \cdot$ Fuminori Miyoshi $^{1}$. \\ Toshio Kaminou $^{1}$ - Shinsuke Kato ${ }^{3}$ - Hisao Ito ${ }^{4}$. Toshihide Ogawa ${ }^{1}$
}

Published online: 7 April 2017

(C) Springer-Verlag Berlin Heidelberg 2017

Erratum to: Neuroradiology

DOI: $10.1007 / \mathrm{s} 00234-013-1199-9$

\section{Originally published in:}

Neuroradiology 2013;55(8):947-953. doi: 10.1007/s00234013-1199-9

Correlation between pathology and neuromelanin MR imaging in Parkinson's disease and dementia with Lewy bodies

Kitao S, Matsusue E, Fujii S, Miyoshi F, Kaminou T, Kato S, Ito H, Ogawa T. and ECR 2013/C-1246 doi:10.1594 /ecr2013/C-1246

The online version of the original article can be found at http://dx.doi. org/10.1007/s00234-013-1199-9

Shinichiro Kitao

sskitao@yahoo.co.jp

1 Division of Radiology, Department of Pathophysiological and Therapeutic Science, Faculty of Medicine, Tottori University, 36-1 Nishi-cho, Yonago, Tottori 683-8504, Japan

2 Department of Radiology, Tottori Prefectural Central Hospital, 730 Ezu, Tottori, Tottori 680-0901, Japan

3 Division of Neuropathology, Department of Brain and Neurosciences, Faculty of Medicine, Tottori University, 36-1 Nishi-cho, Yonago, Tottori 683-8504, Japan

4 Division of Organ Pathology, Department of Microbiology and Pathology, Faculty of Medicine, Tottori University, 36-1 Nishi-cho, Yonago, Tottori 683-8504, Japan
Correlation between pathology and neuromelanin MR imaging in Parkinson's disease and dementia with Lewy bodies Kitao S, Matsusue E, Fujii S, Miyoshi F, Kaminou T, Ogawa T

\section{Notice of redundant publication in:}

Neuroradiology 2013;55(8):947-953

On behalf of all authors, I would like to confirm that our article, "Correlation between pathology and neuromelanin MR imaging in Parkinson's disease and dementia with Lewy bodies," published in Neuroradiology (Neuroradiology 2013;55(8):947-953. doi: 10.1007/s00234-013-1199-9) was based on a study presented and published earlier as EPOS of ECR 2013 (ECR 2013/C-1246 doi:10.1594/ecr2013/C-1246).

Our conclusions derived from the same materials are not different in both publications. However, there are several differences in both publications. First, I want to stress that different analytical methods are used in both publications. In Neuroradiology, we measured the density of the neuromelanin-containing neurons in substantia nigra pars compacta $(\mathrm{SNc})$, while we measured the number of the neuromelanin-containing neurons in SNc in EPOS. This is a fundamentally different and important point. Therefore, their measurement values are different. The regression lines and correlation coefficients between these values and contrast ratios obtained from neuromelanin MR imaging understandably differ. Second, we adopt the Kruskal-Wallis test with Tukey post-hoc analysis as a statistical analysis in EPOS, though this method is not adopted in Neuroradiology. Third, the publication in Neuroradiology refers to the reason why some degree of discrepancy between the density of the neuromelanin- 
containing neurons in $\mathrm{SNc}$ and the contrast ratios obtained from neuromelanin MR imaging in SNc is observed in the case of DLB. Fourth, the publication in Neuroradiology refers to the modified Hoehn and Yahr Scale and the total score of the motor examination section of the Unified Parkinson
Disease Rating Scale at the time of life of PD and DLB cases. Furthermore, neuropathological findings for Alzheimer pathology in all cases are evaluated in Neuroradiology. Last, reference 9 in Neuroradiology is different from EPOS in terms of DLB neuropathological criteria. 